\title{
VARIACIÓN ESPACIO-TEMPORAL DE LAS CIANOPROCARIOTAS DEL ANTIGUO DELTA DEL RÍO SINÚ, CÓRDOBA, COLOMBIA
}

\author{
TIME AND SPACE VARIATION OF CYANOPROKARYOTES FROM THE OLD DELTA SINÚ \\ RIVER, CÓRDOBA, COLOMBIA
}

Martha Mogollón, María Paulina Aycardi, Jorge Galeano, Javier Villalobos y Carolina Arango

\begin{abstract}
RESUMEN
En siete sectores del antiguo delta del río Sinú y en diferentes momentos hidrológicos se evaluaron algunas características limnológicas y se identificaron las cianoprocariotas presentes en el sistema, atendiendo a sus caracteres morfológicos y niveles de organización. Los resultados fueron analizados considerando las diferencias ambientales. Se identificaron un total de 49 morfoespecies, distribuidas en 4 órdenes, 8 familias y 14 géneros entre los que se destacan: Microcystis, Merimopedia, Dolichospermum, Aphanocapsa, Anabaenopsis, Lyngbya, Oscillatoria, Pseudanabaena, Stigonema, Gomphosphaeria y Planktolyngbya, y cuya representación varía según el gradiente de salinidad y la concentración de nitratos y amonio en el medio.
\end{abstract}

PALABRAS CLAVES: cianoprocariotas, delta, río Sinú

\begin{abstract}
In seven sectors of the old delta Sinú River and at different hydrological times some limnological characteristics were evaluated and there were identified cyanoprokariotes that were present in the system, taking into account morphological characteristics and organizational levels. The results were analyzed taking into account their environmental differences. A total of 49 morphospecies were identified, distributed in 4 orders, 8 families and 14 genera such as: Microcystis, Merimopedia, Dolichospermum, Aphanocapsa, Anabaenopsis, Lyngbya, Oscillatoria, Pseudanabaena, Stigonema, Gomphosphaeri and Planktolyngbya and this representation varies according to the salinity gradient, nitrates concentration and ammonium in the system.
\end{abstract}

KEYWORDS: cyanoprokaryotes, delta, Sinú river

\section{INTRODUCCIÓN}

En la región del Caribe colombiano se ubican varios estuarios y lagunas costeras entre las cuales se encuentran el antiguo delta del río Sinú (ADRS) y la Bahía de Cispatá en el Golfo de Morrosquillo. Esta zona estuarina se incluye dentro de los cinco bosques de manglar más extensos y mejor desarrollados del litoral Caribe colombiano (Álvarez-León y Polanía, 1996) y es considerada, por su extensión y grado de conservación, como una de las más importantes de Colombia (Sánchez-Páez et al., 2005).
Durante décadas se ha observado una importante contaminación en esta zona por agroquímicos y uso de pesticidas y fertilizantes, que se refleja con mayor intensidad en los distritos de riego localizados en el medio y bajo Sinú, los cuales vierten sus aguas directamente al río y posteriormente al golfo de Morrosquillo sin ningún tipo de tratamiento (INVEMAR, 2007). Una importante fuente de contaminación es el exceso de nutrientes, especialmente fosfatos y nitrógeno, provenientes principalmente de aguas residuales tratadas de manera insuficiente, residuos agrícolas, abonos y otros desechos de industrias de ganado (Briand et al., 2003). 
Como consecuencia de esto existen cambios ecológicos que inciden en la comunidad fitoplanctónica, la cual refleja de manera rápida cambios en el sistema (Esteves, 1988). La eutrofización también favorece el crecimiento masivo de cianoprocariotas, algunos de cuyos representantes son capaces de producir potentes toxinas con graves repercusiones en la salud pública y en la sanidad animal (Roset et al., 2001).

Las cianotoxinas más comunes en los episodios de toxicidad debido a las cianoprocariotas son las microcistinas (hepatotoxinas), las cuales han sido ampliamente estudiadas y se conoce su resistencia a los tratamientos convencionales de aguas potables, como la cloración, llegando a causar graves problemas sanitarios y medioambientales, incluso a muy bajas concentraciones. $\mathrm{Su}$ presencia se asocia fundamentalmente a especies de Microcystis, Dolichospermum, Aphanizomenom, Nodularia y Planktothrix (Briand et al., 2008; Boaru et al., 2006).

Sin embargo, a pesar de que se ha detectado una tendencia progresiva hacia el aumento de las cianoprocariotas en el antiguo delta del río Sinú, los estudios sobre la presencia y dinámica se este grupo en este sistema son incipientes y se basan principalmente en diagnósticos y evaluaciones ecológicas enfocadas a manglares, peces, fitoplancton y fauna silvestre (INVEMAR,1999; Estela y López, 2005; Ruiz-Ochoa et al., 2008), planes de seguimiento y monitoreo de la zona deltaico estuarina del río Sinú (INVEMAR y URRA, 2007) y en el plan integral de manejo del DMI Cispatá, La Balsa, Tinajones y Sectores Aledaños (INVEMAR y CVS, 2010).

Dada la problemática que supone el impacto al que están expuestas las zonas costeras y marinas debido al proceso de eutrofización, el objetivo principal de este estudio fue evaluar la composición taxonómica y abundancia de las cianoprocariotas potencialmente productoras de toxinas en el antiguo delta del río Sinú (ADRS), en una dimensión espacial y temporal.

\section{MATERIALES Y MÉTODOS}

\section{Área De Estudio}

El área estuarina del ADRS se encuentra localizada en el extremo sur del Golfo de Morrosquillo, entre los $75^{\circ} 46^{\prime}$ y $75^{\circ} 56^{\prime} \mathrm{W}$ y en el paralelo $9^{\circ} 26^{\prime} \mathrm{N}$ de la costa Atlántica Colombiana, con un clima subhúmedo seco y precipitación anual de $1000 \mathrm{~mm}$. Tiene una superficie aproximada de $130 \mathrm{~km}^{2}$, corresponde a las jurisdicciones municipales de San Antero, San Bernardo del viento, y Lorica, departamento de Córdoba (CVS, 1989). Limita con el mar Caribe en su costado norte, en el golfo de Morrosquillo, por el sur con la zona del Distrito de riego La Doctrina, por el este con las estribaciones de la Loma Algarrobo y la Bahía de Cispatá, hasta Playa Blanca y por el oeste con el río Sinú (Figura 1).

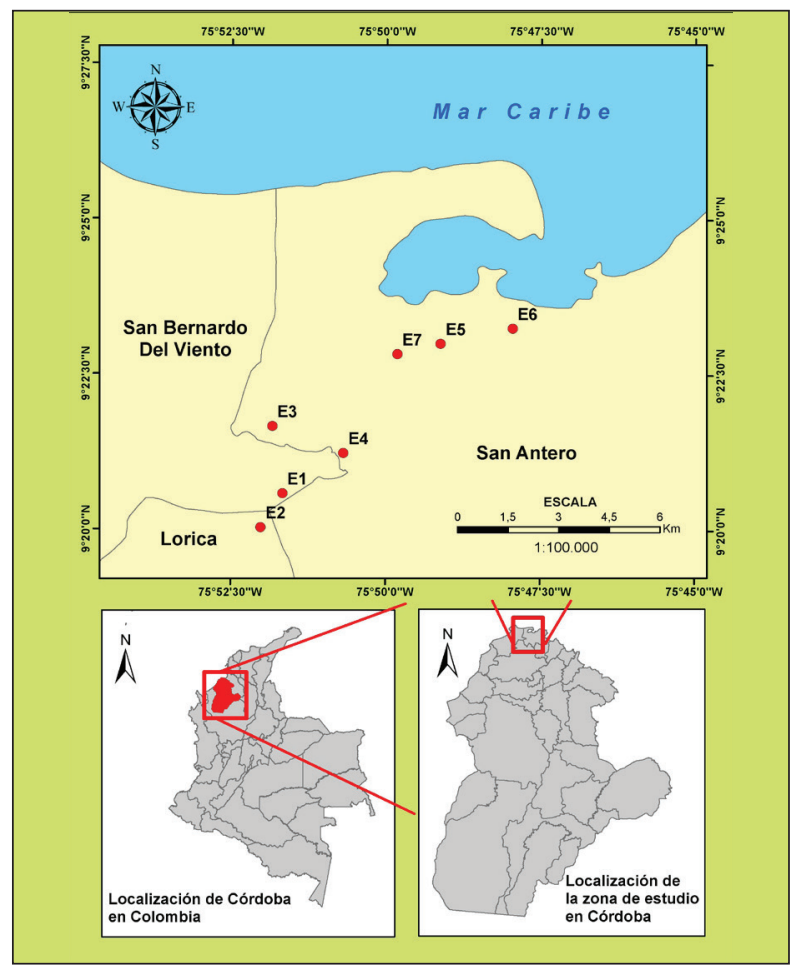

Figura 1. Ubicación geográfica de la bahía de Cispatá, representando los sitios de muestreo: Soledad uno (E1), Soledad dos (E2), Soledad tres (E3), Tijó (E4), Espejo (E5), Bahía (E6), Palermo (E7). Fuente Cartográfica: SIG-OT.

El clima de la zona es cálido y húmedo, la temperatura varía entre 28 y $33^{\circ} \mathrm{C}$ (INVEMAR, 2003). La zona está sometida al régimen de vientos alisios que afectan el Caribe colombiano y que definen las épocas seca y húmeda. En época seca soplan con velocidad variable pero elevada y de manera constante. Durante la época húmeda los vientos son muy variables tanto en dirección como en fuerza y se caracterizan por su mayor porcentaje en calma.

El funcionamiento del sistema lagunar está modulado por las corrientes marinas, la variabilidad anual e 
interanual de la precipitación, los caudales de la cuenca del río Sinú y los desbordes de sus aguas. Por lo cual, se conforman las llanuras litoral y deltaica actuales que dan origen a marismas, manglares, ciénagas y diques a lo largo del antiguo cauce del río Sinú, y de caños como Salado, Sicará, Grande, el Soldado, Remediapobres y los canales de drenaje del distrito de riego de la Doctrina, quienes aportan agua del río a la zona (INVEMAR 2007).

Actualmente solamente llegan al estuario los aportes continentales de agua dulce por la ciénaga de Soledad al desembocar en ésta los caños Sicará, el canal de desagüe del sistema de riego de La Doctrina y por escorrentía de la Cuchilla de Cispatá. La ciénaga de Soledad constituye la cabecera del estuario, receptáculo en su parte oeste de los aportes sedimentarios continentales que están causando la pérdida de fondo en esta área (Aguilera, 1986; Leyton y Delgado, 1992 En: Sánchez-Páez y Álvarez-León, 1997).

\section{Toma De Muestras}

En el ADRS se realizaron cuatro muestreos durante diferentes períodos hidrológicos, los cuales correspondieron a: aguas bajas (diciembre), aguas altas (mayo y junio) y periodo de transición (noviembre), en una serie anual entre 2008 y 2010. Se establecieron siete estaciones teniendo en cuenta aspectos como descarga de los efluentes, productos de las industrias piscícolas y camaroneras y gradiente de salinidad: E1 (Soledad1), E2 (Soledad2), E3 (Soledad3), E4 (Tijó), E5 (Espejo), E6 (Bahía), E7 (Palermo) (Figura 1). En cada estación se tomó una muestra integrada en la columna de agua con una botella tipo Van Dorn y se realizaron arrastres superficiales con una red cónica con poro de 33 micras, evitando así la colmatación de la misma.

Las cianoprocariotas fueron cuantificadas siguiendo la técnica de sedimentación Utermölh (1958). Las muestras fueron examinadas en microscopio invertido (ADVANCE OPTICAL, XDS 1) con una magnificación de 100X, considerando las agremiaciones como una unidad. Posteriormente se verificó la curva de rarefacción. La identificación se realizó teniendo en cuenta características morfológicas de las células, la presencia o no de mucílago y de estructuras especializadas (vesículas de gas, aquinetos, heterocitos y necridios), la forma celular (redonda, oval, alargada), color (verdeazul, verde oliva, rojo, amarillo, marrón) y tamaño celular (grande, mediano, pequeño), y bajo los criterios de identificación de Komárek y Anagnostidis (1998), Komárek y Komárková (2004), Komárek y Anagnostidis (2005) y Bicudo (2006). Se tomaron microfotografías con cámara Olympus DP71 y la medidas respectivas con ayuda de los programas DPcontroller y del Sofware analizador de imagen Carl Zeiss, Axiovision.

\section{Variables Hidrológicas}

En cada estación se evaluaron las siguientes variables físicas y químicas: oxígeno disuelto $(\mathrm{mg} / \mathrm{L})$, porcentaje de saturación de oxígeno, $\mathrm{pH}$, temperatura $\left({ }^{\circ} \mathrm{C}\right) \mathrm{y}$ salinidad, utilizando un YSI 55 Standard Methods 4500OG, transparencia del agua (m), utilizando disco Secchi de $15 \mathrm{~cm}$ de diámetro. Los nutrientes como ortofosfatos $\left(\mathrm{PO}_{4}^{-3}\right)$, nitratos $\left(\mathrm{NO}^{3-}\right)$ y nitritos $\left(\mathrm{NO}^{2-}\right)$ se midieron siguiendo los protocolos establecidos en el Standard Methods (APHA -AWWA-WPCF, 2005) y utilizando un espectrofotómetro HACH modelo DR/2400.

\section{Procesamiento y Análisis de la Información}

Para establecer los posibles patrones de distribución espacial, a partir de los valores de riqueza se empleó el análisis de similaridad entre los diferentes puntos de muestreos CLUSTER del programa PLYMOUTH ROUTINES IN MULTIVARIATE ECOLOGICAL RESEARCH V5 (PRIMER v5) (Carr, 1996), aplicando los índices de Jaccard. Para comprobar el supuesto de normalidad de los datos, se aplicó la prueba de Shapiro-Wilk y posteriormente se realizó un Análisis de Varianza (ANDEVA) no paramétrica, con el fin de comparar las diferencias significativas entre estaciones y períodos hidrológicos, utilizando el programa XLSTAT 2011.

\section{RESULTADOS}

\section{Variables Físicas y Químicas}

El valor promedio de transparencia del agua para el período de muestreo fue de $0,57 \mathrm{~m}$, encontrándose una clara diferenciación entre las épocas climáticas, con los mayores valores en aguas bajas debido posiblemente al menor arrastre de sedimento y menor resuspensión de sedimentos (Tabla 1). Se evidenció un gradiente espacial, presentándose los valores mínimos en los sectores con mayor flujo como los caños y los máximos valores en sectores con mayor cercanía a la zona marina como la bahía de Cispatá (0,89 m). 
Tabla 1. Concentración de nutrientes en el antiguo delta del río Sinú

\begin{tabular}{|c|c|c|c|c|c|c|c|c|c|}
\hline \multirow{2}{*}{ MUESTREOS } & \multirow{2}{*}{ ESTACIONES } & \multirow{2}{*}{$\frac{\text { Transparencia }}{\text { (m) }}$} & \multirow{2}{*}{$\begin{array}{c}\text { Oxígeno } \\
\text { Disuelto }\end{array}$} & \multirow[t]{2}{*}{$\mathrm{pH}$} & \multirow{2}{*}{$\begin{array}{c}\text { Temperatura } \\
{ }^{\circ} \mathrm{C}\end{array}$} & $\mathrm{NO}_{2}$ & $\mathrm{NO}_{3}$ & $\mathrm{NO}_{4}$ & $\mathrm{PO}_{4}$ \\
\hline & & & & & & \multicolumn{4}{|c|}{$(\mathrm{mg} / \mathrm{L})$} \\
\hline \multirow{5}{*}{ 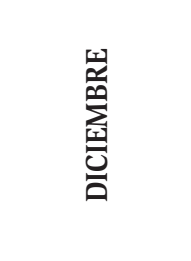 } & Soledad * & 0,71 & 2,3 & 8,5 & 29,5 & 0,01 & 0,14 & 0,55 & 0,05 \\
\hline & Palermo & 0,85 & 2,5 & 8,6 & 29,7 & 0,03 & 0,11 & 0,77 & 0,07 \\
\hline & Tijó & 0,60 & 2,7 & 7,9 & 30,1 & 0,04 & 0,08 & 0,7 & 0,08 \\
\hline & Espejo & 0,53 & 6,5 & 7,8 & 30,8 & 0,02 & 0,07 & 0,69 & 0,08 \\
\hline & Bahía & 0,54 & 7,6 & 8,2 & 30,0 & 0,01 & 0,05 & 0,06 & 0,013 \\
\hline \multirow{5}{*}{ 芳 } & Soledad * & 0,61 & 1,2 & 7,3 & 31,0 & 0,01 & 0,15 & 0,71 & 0,034 \\
\hline & Palermo & 0,62 & 1,8 & 7,7 & 31,0 & 0,04 & 0,12 & 0,66 & 0,050 \\
\hline & Tijó & 0,50 & 1,1 & 7,3 & 31,4 & 0,1 & 0,07 & 0,89 & 0,008 \\
\hline & Espejo & 0,90 & 5,8 & 7,4 & 31,0 & 0,05 & 0,07 & 0,05 & 0,009 \\
\hline & Bahía & 0,89 & 6,9 & 7,5 & 32,0 & 0,01 & 0,07 & 0,05 & 0,012 \\
\hline \multirow{5}{*}{ 点 } & Soledad * & 0,60 & 2,3 & 7,7 & 31,6 & 0,01 & 0,16 & 0,65 & 0,05 \\
\hline & Palermo & 0,35 & 3,2 & 7,4 & 30,7 & 0,03 & 0,11 & 0,92 & 0,04 \\
\hline & Tijó & 0,48 & 2,7 & 7,5 & 30,5 & 0,09 & 0,09 & 0,77 & 0,011 \\
\hline & Espejo & 0,58 & 6,2 & 7,9 & 32,5 & 0,02 & 0,01 & 0,05 & 0,012 \\
\hline & Bahía & 0,54 & 7,0 & 8,0 & 31,2 & 0,01 & 0,06 & 0,04 & 0,011 \\
\hline \multirow{5}{*}{$\sum_{\sum}^{0}$} & Soledad * & 0,52 & 0,9 & 6,7 & 30,2 & 0,01 & 0,12 & 0,77 & 0,051 \\
\hline & Palermo & 0,48 & 2,2 & 7,0 & 32,6 & 0,06 & 0,11 & 0,83 & 0,06 \\
\hline & Tijó & 0,59 & 2,0 & 7,1 & 32,6 & 0,09 & 0,08 & 0,86 & 0,014 \\
\hline & Espejo & 0,72 & 5,1 & 7,3 & 32 & 0,09 & 0,01 & 0,01 & 0,014 \\
\hline & Bahía & 0,52 & 5,0 & 7,3 & 33 & 0,01 & 0,07 & 0,06 & 0,015 \\
\hline
\end{tabular}

* Concentración promedio de las estaciones Soledad 1, Soledad 2 y Soledad 3.

La salinidad máxima (34 ppt) se registró en la estación Bahía y en general en las estaciones más próximas al mar, asociado a la mayor presencia de sales disueltas. Los mínimos valores (9) corresponden a la estación Soledad 1. En general se mantuvo una diferenciación entre las épocas de aguas altas y aguas bajas, coincidente con los aportes hídricos de cada una de las épocas.

La temperatura varió entre $25^{\circ} \mathrm{C}$ y $33{ }^{\circ} \mathrm{C}$, con una tendencia homogénea espacial y temporal. Los valores de oxígeno disuelto en el sistema lagunar variaron entre 0,7 mg.l-1 y 7,61 mg.l-1. Los sectores internos como Soledad y Caño Palermo presentaron la más bajas concentraciones de toda el área, siendo estas zonas de constante resuspensión de sedimentos, mientras que los valores más altos se presentaron en las estaciones con mayor mezcla como Espejo y Bahía.

El pH presentó valores homogéneos con una leve disminución en la época de aguas altas asociado al incremento del aporte hídrico. El sector de Soledad presentó el valor promedio más alto $(7,6)$.

Los nutrientes (fosforo y nitrógeno), presentaron bajas concentraciones, en algunos casos hasta indetectables (0,01 mg/L). Las formas principales de nitrógeno fueron los nitratos y el amonio. Los nitratos presentaron concentraciones entre $0,16 \mathrm{mg} / \mathrm{L}$ y $0,01 \mathrm{mg} / \mathrm{L}$, mientras 
que el amonio presentó niveles más elevados, con concentraciones cercanas a $1 \mathrm{mg} / \mathrm{L}$ (Tabla 1 ). Las concentraciones más altas de nitratos se presentaron en las estaciones con menores niveles de salinidad (Soledad y Tijó), dichos puntos suelen relacionarse con la contaminación orgánica de origen humano. Las concentraciones de estas variables fueron menores en aguas altas que en aguas bajas. El ortofosfato presentó los valores más altos en el sector Soledad y Palermo, notándose entre épocas climáticas un incremento para aguas altas.

Las principales diferencias en el sistema estuvieron representadas por la salinidad y los nutrientes, las estaciones más internas presentaron los menores valores de transparencia y salinidad, así como las mayores concentraciones de nutrientes.

Tabla 2. Cianoprocariotas registrados en el antiguo delta del río Sinú

\begin{tabular}{lllc}
\hline \multirow{2}{*}{ ORDEN } & \multicolumn{1}{c}{ FAMILIA } & \multicolumn{1}{c}{ NÚMERO DE } \\
MORFOESPECIES
\end{tabular}

\section{CIANOPROCARIOTAS}

Se registraron un total de 49 morfoespecies de cianoprocariotas distribuidas en 3 órdenes, 7 familias y 15 géneros. En su mayoría pertenecientes a las familias Oscillatoriaceae (25\%), Nostocaceae (23\%), Phormidiaceae (18\%) y Merismopediaceae (14\%). El género Oscillatoria presentó el mayor número de morfoespecies al igual que los géneros cf Dolichospermum y Spirulina (Figura 2), las cuales presentaron especies productoras de citotoxinas. Se registraron otros géneros con menor riqueza pero de igual importancia toxicológica como cf Microcystis, Merimopedia, Aphanocapsa, Anabenopsis, Lyngbya y Chroococcus (Figura 3).

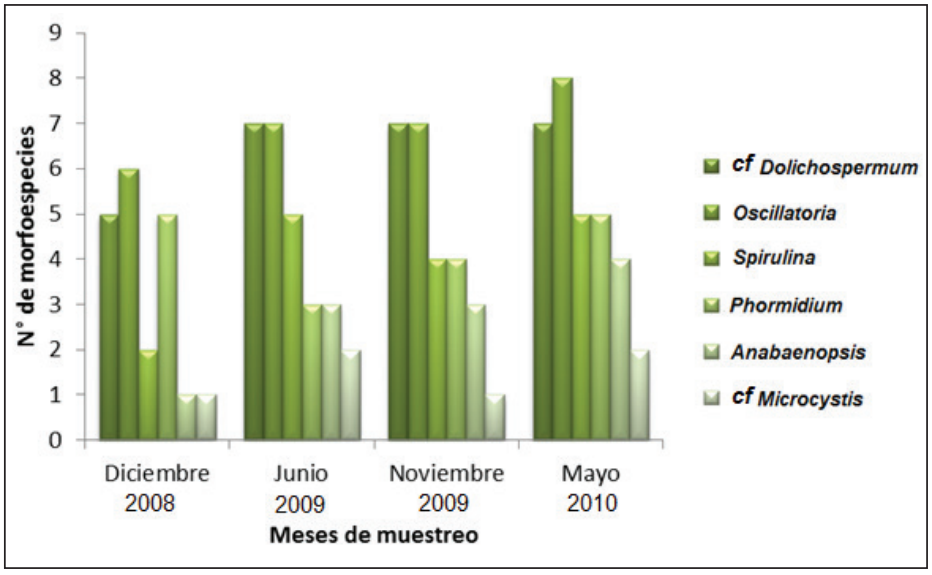

Figura 2. Representación de los géneros con mayor riqueza en el antiguo delta del rio Sinú durante los diferentes periodos de muestreos. 

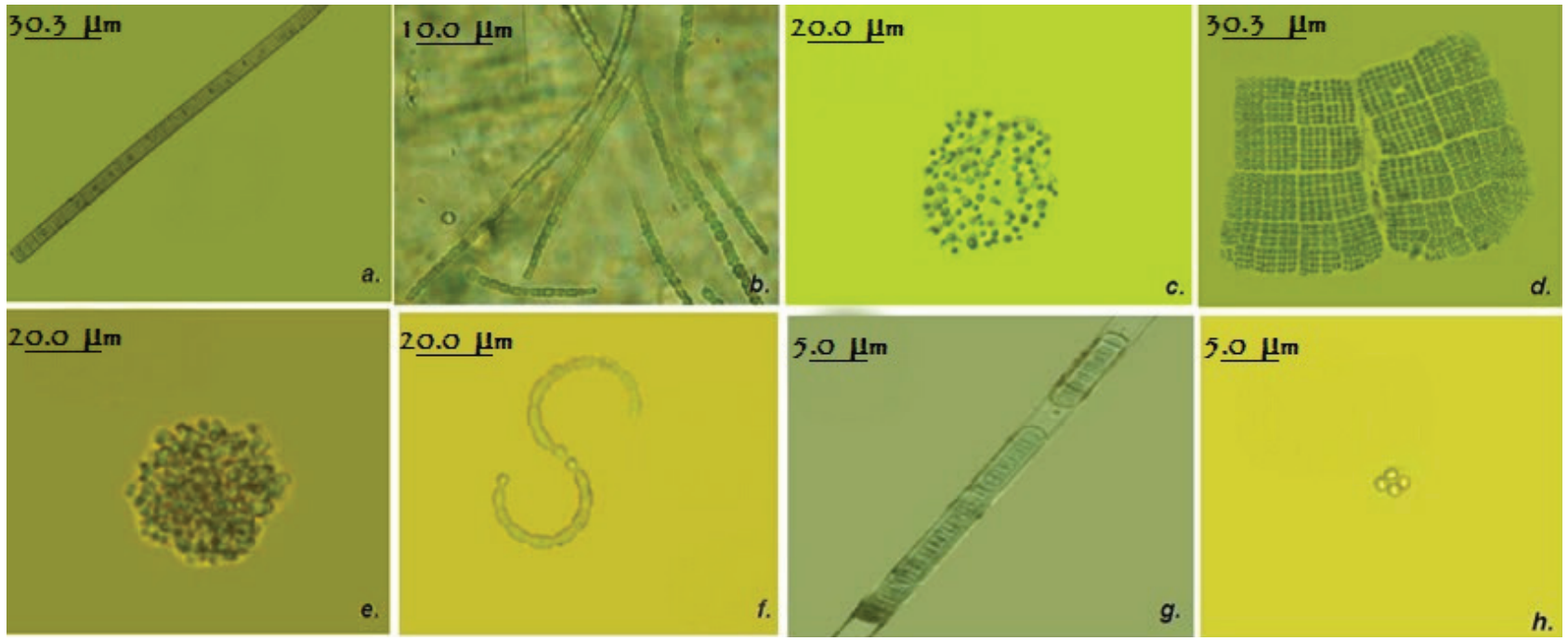

Figura 3. a. Oscillatoria; b. cf Dolichospermum; c. cf Microcystis; d. Merimopedia; e. Aphanocapsa; f. Anabenopsis; g. Lyngbya; h. Chroococcus.

En la época de transición (noviembre) se reportó el mayor número de morfoespecies (36), seguido del periodo de lluvias (junio) con 31 morfoespecies. Para la época seca (diciembre) se encontró el menor número de cianoprocariotas (24).

Los géneros cf Dolichospermum y Oscillatoria presentaron el mayor número de morfoespecies a lo largo de todas las estaciones de muestreo (Figura 4). Las mayores riquezas se registraron en las estaciones Tijó y Bahía (31 morfoespecies), representado en su mayoría por dichos géneros. Las menores riquezas fueron registradas en las estaciones Espejo, Soledad 1 y Soledad 3 (28, 24 y 23 morfoespecies).

Se observó la formación de dos grupos con alta similaridad en cuanto a las morforspecies presentes en el sistema, asociándose las estaciones Soledad y Palermo (Grupo I), Espejo y Tijó (Grupo II), alejándose del grupo la estación Bahía, zona que presentó también diferencias en cuanto a sus características hidrológicas (altos valores de salinidad y menores concentraciones de las fuentes nitrogenadas), lo que determinó la presencia de las cianoprocariotas en este sector (Figura 5).

Durante todos los periodos de muestreo las formas filamentosas como Planktolyngbya, Anabaenopsis y Spirulina presentaron la mayor abundancia,

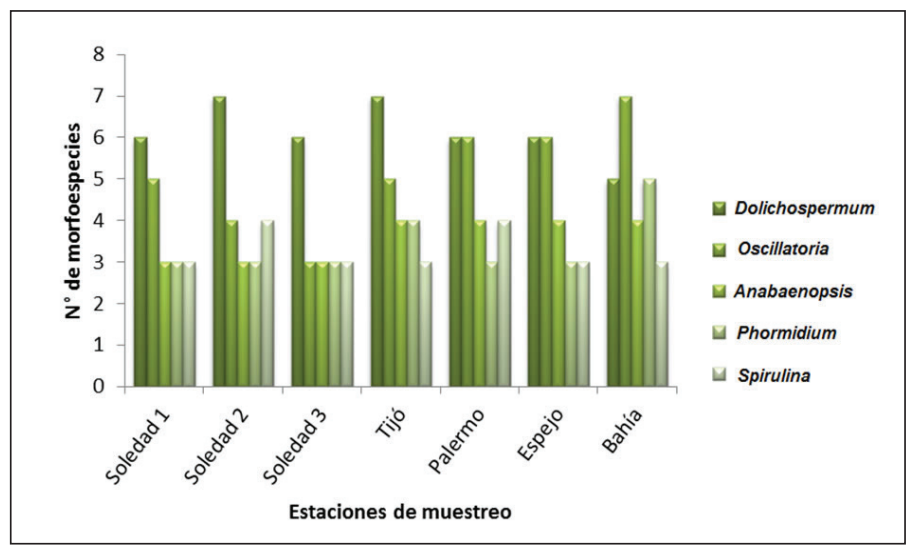

Figura 4. Representación de los géneros con mayor riqueza en el antiguo delta del rio Sinú en las estaciones de muestreo.

resaltando el dominio de agregados celulares como Chroococcus. En la época de aguas bajas (diciembre) P. limnetica presentó la mayor abundancia, seguida de Chroococcus sp1, y otras menos abundantes como Spirulina sp2 y Dolichospermum sp4 (Figura 6). En las estaciones Soledad 1, 2 y 3, P. limnetica presentó las mayores abundancias, seguido por Anabaenopsis sp1 y Spirulina sp2. (Figura 7). En Palermo, Espejo y Bahía la tendencia se mantuvo con la dominancia de P. limnetica, resaltando su dominancia en la estación de Bahía.

El análisis estadístico indicó diferencias significativas en cuanto a la abundancia de las cianoprocariotas entre las estaciones de muestreo $(\mathrm{p}<0,05)$, registrándose los mayores valores en los 
sectores dulceacuícolas (Figura 8). Entretanto en ambientes con reducida transparencia como Soledad, Palermo y Tijó se destacó la presencia y dominio de cianoprocariotas con morfotipos atenuados, como las especies filamentosas con capacidad de lograr una posición óptima en la columna de agua, incrementando su área de captación de luz y nutrientes.

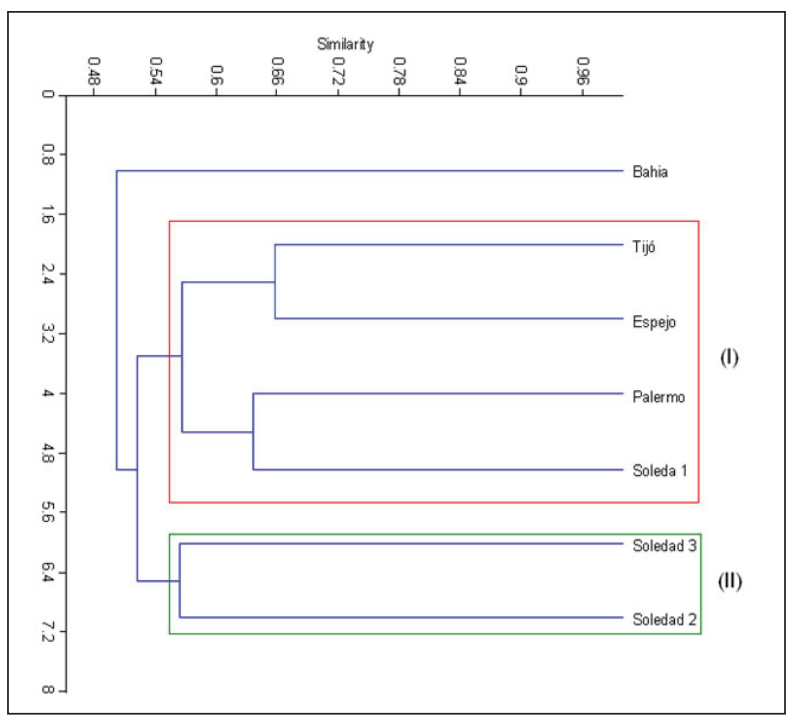

Figura 5. Cluster de similitud según la riqueza $\left(\mathrm{N}^{\circ}\right.$ de especies / estaciones) obtenidas mediante el índice de asociación de Jaccard, en las estaciones y periodos de muestreo en el antiguo delta del rio Sinú.

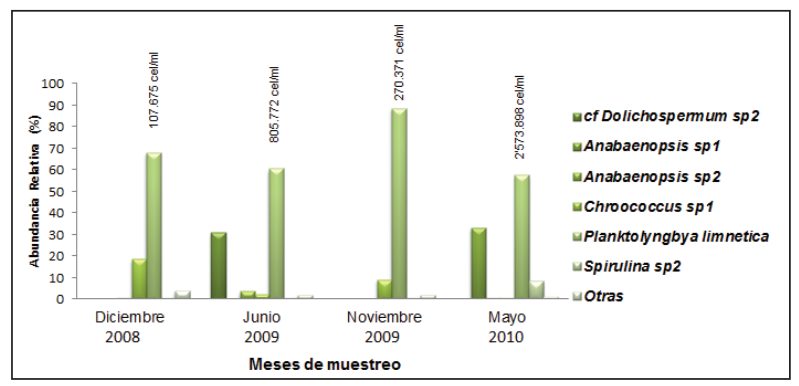

Figura 6. Géneros con mayor abundancia en el antiguo delta del rio Sinú durante los diferentes períodos de muestreo.

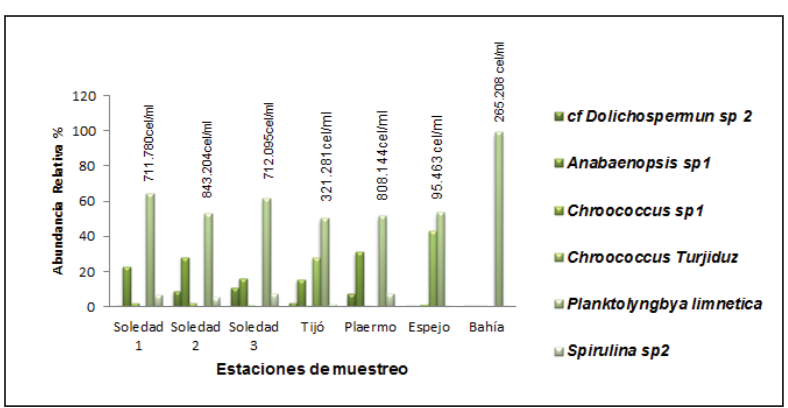

Figura 7. Géneros con mayor abundancia en el Antiguo delta del rio Sinú en las estaciones de muestreo.

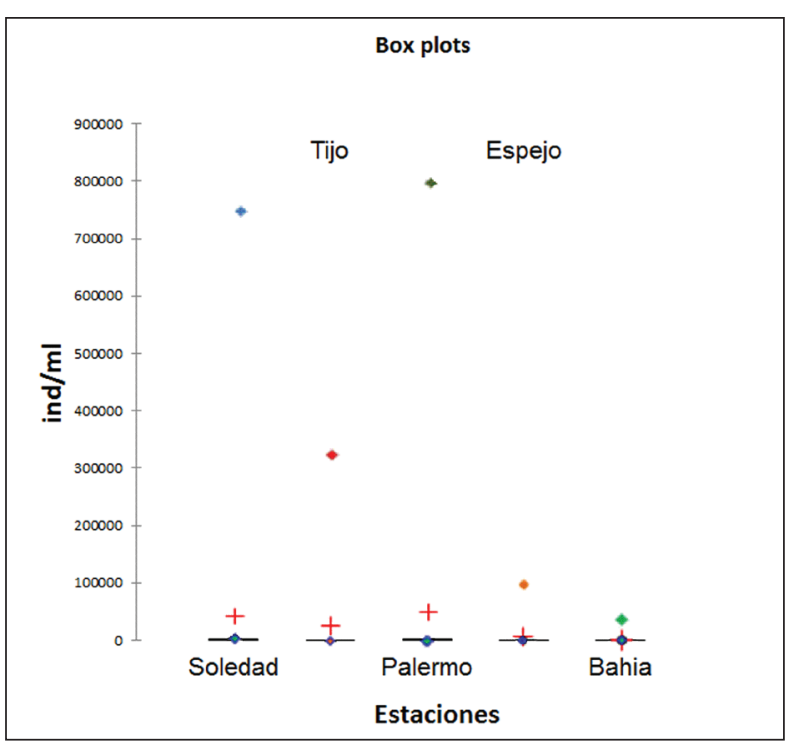

Figura 8. Variación espacial de la abundancia de las cianoprocariotas en el antiguo delta del rio Sinú.

\section{DISCUSIÓN}

Dentro de los grupos filamentosos dominantes en el ADRS se registraron individuos del género Oscillatoria, reportado por la literatura como un grupo con varias especies productoras de toxinas (Dow y Swoboda, 
2000; Edwards et al., 2004), el cual se presenta en el sistema como el género de mayor riqueza en todos los periodos hidrológicos, excepto en época de aguas bajas. Lo anterior posiblemente este asociado a la disminución de los aportes de aguas dulceacuícolas provenientes del río, permitiendo en su lugar el dominio de otros géneros que logran adecuarse a las nuevas condiciones. Otros géneros con menor riqueza pero de igual importancia toxicológica fueron: cf Dolichospermum, cf Microcystis, Merimopedia, Aphanocapsa, Anabaenopsis, Lyngbya, Pseudoanabaena (Yunes et al., 2007).

Al analizar en conjunto las variaciones de abundancia de las cianoprocariotas en la escala espacio-tiempo, se puede inferir que este obedece al gradiente de salinidad que se presenta en el sistema, la cual aumenta en la medida de que las estaciones presentan mayor relación con el mar. De igual forma, las mayores abundancias se asocian a los sectores con mayor concentración de nutrientes (Soledad, Palermo y Tijó) en especial de nitrato y nitrógeno amoniacal, producto de los vertimientos del distrito de riego y de las actividades acuícolas que se asocian a estos sectores, reflejándose en los cambios de las variables químicas a través del tiempo (INVEMAR, 2007). Lo anterior favorece el crecimiento de las cianoprocariotas, en particular de aquellas formas filamentosas que crecen a bajas intensidades lumínicas, generándose un proceso de retroalimentación en cuanto a la dominancia de las cianobacterias al restringir con su elevado crecimiento aún más el campo lumínico (Carey et al., 2012). La baja trasparencia en las estaciones de Soledad favorece el crecimiento de cianobacterias filamentosas como Planktolyngbya, las cuales pueden utilizar la energía lumínica en rangos del espectro que no son utilizados por otros grupos (Jeffrey et al., 1997). Además su estructura pigmentaria les confiere una alta tolerancia a las condiciones de penumbra (UNESCO, 2009). Otro aspecto relevante que contribuye a dominio de las cianoprocariotas está asociado a su plasticidad morfológica incidiendo en el establecimiento de sus poblaciones (UNESCO, 2009).

En este tipo de ecosistema el consumo de oxígeno en el sedimento y en el agua de fondo conduce a la anoxia, especialmente bajo condiciones de estratificación de la columna de agua en verano (Paerl, 1988). Esto interfiere en los procesos de nitrificación de algunas formas de nitrógeno, permitiendo la acumulación de amonio que es rápidamente utilizado por las algas y cianoprocariotas. A su vez, la anoxia promueve una serie de mecanismos físicos y químicos que culminan con la liberación de fosfato de los flóculos de hidróxido de hierro que lo mantenían retenido en el sedimento bajo condiciones de oxigenación (Kalff, 2002). Esta carga de fósforo contribuye a estimular aún más la producción primaria y fomentar las floraciones de cianoprocariotas. Así, puede producirse una situación de perpetuación a largo plazo debido a esta retroalimentación positiva entre las causas y las consecuencias del fenómeno, posibilitando incluso presencia constante de floraciones de cianoprocariotas, como se evidencia en este estudio. Sistemas con alta carga de nutrientes conducen gradualmente al incremento de la productividad, lo que a su vez, provoca la reducción de la biodiversidad, crecimiento desmedido de plantas acuáticas, malos olores por falta de oxígeno, mortandad de peces, deterioro general de la calidad de agua y la dominancia progresiva de ciertas especies oportunistas, incluyendo cianobacterias (Conde, 2009 En: UNESCO, 2009).

En sistemas como el ADRS, que presenta en su ciénagas internas como Soledad baja transparencia y alta carga de nutrientes, es evidente el dominio de cianoprocariotas con rasgos morfológicos atenuados y filamentosos que les confieren propiedades que les permite ocupar posiciones optimas en la columna de agua, aumentado su área de captación de luz y nutrientes (Visser et al., 2005). De igual forma, su capacidad de fijar nitrógeno atmosférico y/o de regular su flotabilidad mediante vacuolas de gas les permite desplazar a las algas eucariotas y dominar en el sistema (Arrigo, 2005). La baja presencia de cianoprocariotas heterocistadas en el sistema posiblemente está relacionada con la alta concentración de fuentes nitrogenadas disponibles. El aumento en los niveles de caudal (INVEMAR-URRA 2007) es otro factor que ha propiciado un incremento en la densidad de cianoprocariotas en las estaciones estuarinas y marinas, debido al gran aporte de nutrientes.

En referencia a la estructura de la comunidad fitoplanctónica existen diferencias en términos de abundancia a nivel espacial; asociadas a la entrada de agua dulce al sistema. Los resultados sugieren un favorecimiento a poblaciones que responden de manera positiva a los altos aportes de materia orgánica y baja transparencia, en la dimensión espacio-tiempo. Esta situación se evidenció en el hecho de que la mayoría de las morfoespecies presentaron un nivel de organización filamentoso.

Durante el estudio se presentaron floraciones de cianoprocariotas; con morfoespecies potencialmente tóxicas. Leflaive y Ten-Hage (2007) indican la posibilidad 
de que las sustancias alelopáticas como las cianotoxinas que segregan las cianoprocariotas, sean el principal motivo de preocupación en los cuerpos de agua, ya que puede representar un problema para los otros organismos del medio acuático y para el ser humano.

La presencia de cianoprocariotas comúnmente menos apetecible para los herbívoros (Reynolds, 2006), resulta en una disminución del alimento y la energía disponible para los niveles tróficos superiores, causando modificaciones importantes de la estructura global del ecosistema, especies relevantes para la estructura trófica o desde el punto comercial pueden ser negativamente impactadas o desaparecer por el proceso de eutrofización (Rodríguez et al., 2001).

Ahora bien, podemos concluir que la entrada de agua dulce y la elevada concentración de nutrientes favorece el mantenimiento en el tiempo las poblaciones de cianoprocariotas, las cuales encuentran las condiciones propicias para su desarrollo, al no variar de forma drástica las condiciones del ecosistema entre los diferentes períodos hidrológicos.

\section{AGRADECIMIENTOS}

El estudio fue realizado en el marco del macroproyecto "Identificación, detección y cuantificación de cianobacterias y microcistinas en el antiguo delta del río Sinú (ADRS), Departamento de Córdoba, Colombia”, liderado y financiado por la Universidad de Córdoba de Colombia y la Universidad del Sinú de Colombia.

\section{REFERENCIAS}

Álvarez-León R. y J. Polanía. 1996. Los manglares del Caribe colombiano: Síntesis de su conocimiento. Revista de la Academia Colombiana de Ciencias Exactas, Físicas y Naturales. Vol 20(78): 447-464.

Aguilera, Q. J. 1986. Clasificación del estuario antiguo cauce del río Sinú. Trabajo de Grado. Escuela Naval "Almirante Padilla", Cartagena. 108 p.

APHA (American Public Health Association), AWWA (American water Works Association) y WEF (Water 100 Environment Federation). 1992. Standard methods for the examination of water and wastewater. 18 ed. U.S.A. 1234 p.

Arrigo, K. R. 2005. Marine microorganisms and global nutrient cycles. Nature 437: 349-55
Bicudo, C. 2006. Cyanophyceae/Cyanobacteria. pp. 58-95. En: Bicudo C. y M. Menezes (Eds.), Gêneros de Algas de Águas Continentais do Brasil. São Carlo, Brasil. Ed. RIMA. Boaru, D. A., N. Drago, M. Welker, A. Bauer, A. Nicoara y K. Schirmer. 2006. Toxic potential of microcystin-containing cyanobacterial extracts from three Romanian freshwaters. Toxicon. 47:925-32.

Briand, J., S. Jacquet, C. Bernard y J. F. Humbert. Health. 2003. Hazards for terrestrial vertebrates from toxic cyanobacteria in surface water ecosystems. Vertebrate Research. 34:361- 377.

Briand E., C. Yépremian, J. F. Humbert y C. Quiblier. 2008. Competition between microcystin and non-microcystinproducing Planktothrix agardhii (Cyanobacteria) strains under different environmental conditions. Environmental Microbiology.10: 3337-48.

Carr, M. R. 1996. Plymouth Routines in Multivariate cological Research. User Manual. Plymouth Marine Laboratory, UK. 41 p.

Corporación Autónoma Regional de los Valles del Sinú y del San Jorge CVS. 1989. Plan de ordenamiento y manejo de la zona de manglar Antiguo delta del Río Sinú. Informe final Vol. II. INCCO Ltda. Bogotá.. 421 p.

Dow, C. S. y U. K. Swoboda. 2000. Cyanotoxins. Pp. 613632 En: B. A. Whitton y M. Potts, (Eds). The Ecology of Cyanobacteria: Their Diversity in Time and Space, Kluwer Academic Publishers, Norwell, Massachusetts, EE.UU. 775 p.

Estela, F. y M. López. 2005. Aves de la parte baja del río Sinú, Caribe colombiano; inventario y ampliacionaes de distribución. Boletín Investigaciones Marinas y Costeras. 34 (1): 7-42.

Esteves, F. S. 1988, Fundamentos de limnología. Interciencia Ltda (Ed). 2 edición, Brasil. 430 p.

INVEMAR-CVS. 2010. Plan Integral de Manejo DMI Cispatá - La Balsa - Tinajones y sectores aledaños. 76 p.

INVEMAR-URRA. 2007. Plan de seguimiento y monitoreo de la zona deltaico estuarina Del río Sinú (noviembre de 2000 - noviembre de 2007). Instituto de Investigaciones Marinas y Costeras “José Benito Vives de Andreis”. 444 p.

INVEMAR. 2003. Plan de Seguimiento y Monitoreo de la Zona Deltaico Estuarina del Río Sinú. Informe Final (noviembre de 2000 a mayo de 2003). Instituto de Investigaciones Marinas y Costeras "José Benito Vives de Andreis". Santa Marta, 473 p. 
INVEMAR. 1999. Diagnostico y evaluacion ecológica del antiguo delta del río Sinú on enfasis en Bahía Cispatá y ciénagas aledañas. Informe final. Instituto de Investigaciones Marinas y costeras, José Benito Vives de Andreis. Santa Marta D.T.

Jeffrey, S. W., R. F. C. Mantoura y S. W. Wright. 1997. Phytoplankton pigments in oceanography. Monographs on oceanographic methodology. Paris, UNESCO: 661 p.

Kalff, J. 2002. Limnology. New Jersey, Prentice-Hall. 592 p.

Komárek, J. y K. Anagnostidis. 1998. Cyanoprokaryota. 1. Teil Chroococcales. En: Ettl, H., G. Gärtner, H. Heynig y D.Möllenhauer (eds.). Sübwasserflora von Mitteleuropa. Gustav Fischer Verlag, Stuttgart, 1 - 548.

Komárek, J y J. Komárková. 2004. Taxonomic review of the cyanoprokaryotic genera Planktothrix and Planktothricoides. Fottea. 4: 1-18

Komárek, J. y K. Anagnostidis. 2005. Cyanoprokaryota. 2. Teil Oscillatoriales. En: B. Büdel, L. Krienitz, G. Gärtner y M. Schagerl (eds). Sübwasserflora von Mitteleuropa. Elsevier: Spektrum Akademischer Verlag, Munique, 759 p.

Paerl, H. W. 1988. Nuisance phytoplankton blooms in coastal, estuarine and inland waters. Limnology and Oceanography, 33(4):823-847.

Reynolds, C.S. 2006. Ecology of phytoplankton. Cambridge, Cambridge University. 550 p.

Rodríguez, C., M. Mancini, C. Prosperi y A. Weyers. 2001. Calidad de agua de una laguna recreacional del centro-oeste de la provincia de Córdoba, Argentina. Revista Aquatic 12 p. Roset, J., S. Aguayo y M. J. Muñoz. 2001. Detección de cianoprocariotas y sus toxinas. Una revisión. Revista de Toxicología. Madrid. 18: 65-71.
Ruiz-Ochoa, M., G. Bernal y J. Polanía. 2008. Influencia del río Sinú y el mar caribe en el Sistema lagunar de Cispatá. Boletin Investigaciones Marinas y Costeras. 37 (1): 31-51.

Sánchez-Páez, H. y R. Álvarez-León. 1997. Diagnóstico y zonificación preliminar de los manglares del Caribe de Colombia. Ministerio del Medio Ambiente, Organización de Maderas Tropicales. Santa Fe de Bogotá, Colombia. 511 p.

Sánchez-Páez, H., G. Ulloa-Delgado, H. Tavera-Escobar y W. Gil-Torres. 2005. Plan de manejo integral de los manglares de la zona de uso sostenible sector estuarino de la bahía de Cispatá departamento de Córdoba. OIMT. CVS. CONIF. Ministerio de Ambiente, Vivienda y Desarrollo Territorial, Bogotá D.C. 202 p.

UNESCO. 2009. Cianobacterias Planctónicas del Uruguay. Manual para la identificación y medidas de gestión. Sylvia Bonilla (ed.). Documento Técnico PHI-LAC, N 16.105 p.

Utermölh, H. 1958. Zur Vervollkommung der guantitativen Phytoplankton - Methodik. Mitt. Internationale Ver. Theoretische und Angewandte Limnologie. 9: 567-596.

Visser, P. M., B. W. Ibelings, L. R. Mur y A. E. Walsby. 2005. The ecophysiology of the harmful cyanobacterium Microcystis: features explaining its success and measures of its control. Pp. 109-142. En: J. Huisman, H. C. P. Matthijs y M. Visser (Eds.) Dordrecth, Netherlands Springer.

Yunes, J., S. De La Rocha, D. Giroldo, S. B. D. Silveira, R. Comin, M. D. Bicho, S. S. Melcher, C. L. Sant' Anna y A. A. Vieira. 2007. Release of carbohydrates and proteins by a subtropical strain of Raphidiopsis brookii (cyanobacteria) able to produce saxitoxin at three nitrate concentrations. Journal of Phycology 45: 585-591.

Fecha de recepción: 20/06/2014

Fecha de aceptación: 15/11/2014

Para citar este artículo: Mogollón, M., M. P. Aycardi, J. Galeano, J. Villalobos y C. Arango. 2014. Variación espacio-temporal de las cianoprocariotas del antiguo delta del río Sinú, Córdoba,

Colombia. Revista Intropica 9: 92 - 101 